\title{
CONCEPTUAL DESIGN OF A DUST MONITOR DIAGNOSTIC FOR ITER
}

\section{Evgeny VESHCHEV ${ }^{1}$}

ITER Organization,

Route de Vinon-sur-Verdon - CS 90046 - 13067 St Paul Lez Durance Cedex - France

E-mail: evgeny.veshchev@iter.org

\section{G. Vayakis, G. De Temmerman, K. Ebisawa, Ph. Gitton, G. Jagannathan, R.} Reichle, P. Shigin, M. Walsh, R. Walton

ITER Organization, Building 81/205, DIP, Directorate for CODAC, Heating and Diagnostics Route de Vinon-sur-Verdon - CS 90046 - 13067 St Paul Lez Durance Cedex - France

ITER requires diagnostics for monitoring of in-vessel dust and concomitant $\mathrm{T}$ accumulation level. To monitor these under the divertor, a dedicated diagnostic referred to as dust monitor is being developed. It will be based on a combined endoscope / dust collector designed to travel approximately $20 \mathrm{~m}$. The diagnostic can use two tools - one for fine viewing of dust with resolution down to a few tens of microns across a few $\mathrm{mm}$ and another one for dust collection. Both endoscopes will have coarse viewing with resolution of few hundred microns over a wider area to allow the possibility for more general inspection of surrounding environment, obstacle avoidance etc. All viewing will be done using radiation hard fibre bundles. The collected dust will be characterized to determine its T-content, elemental composition, mass and size distribution.

First EPs Conference on Plasma Diagnostics - $1^{\text {st }}$ ECPD

14-17 April 2015,

Villa Mondragone , Frascati (Rome) Italy 


\section{Introduction}

ITER is a basic nuclear installation (INB) and as such, safety is the most important driver of key aspects of its design. ITER's license requires that the quantity of dust and Tritium in the vacuum vessel remain below a limit of $1000 \mathrm{~kg}$ for dust and $1 \mathrm{~kg}$ for Tritium. In addition to that, dust may influence plasma performance and might become an obstacle to advanced performance even if its amount is below safety limits. Therefore, measurement of dust and regular cleaning of certain in-vessel regions are important for the successful operation of ITER. Dust inside tokamak will be collected by the Remote Handling Multi-Purpose Deployer (MPD) [1] during long-term maintenance, once every two years. A very conservative estimate of the in-vessel dust inventory might be obtained by periodic monitoring of the ITER First Wall erosion/re-deposition using the In-Vessel Viewing System (IVVS) [2], assuming that all eroded material converts to dust.

Endoscopes are widely used in various areas from medical services to industry for various applications. They allow survey and manipulation in remote areas not accessible to other tools. In ITER endoscopes are planned to be used for inspection of the vacuum vessel welds and for dust monitoring (this paper).

\section{Dust Monitor layout and main components}

Two Dust Monitoring Endoscopes (DMEs) are being developed for ITER, for fine viewing and dust collection for subsequent analysis, respectively. Both endoscopes will use the same infrastructure for access to the vacuum vessel entry points through the isolation valves. Endoscopes will be installed at two toroidal locations through the lower ports (in lower port \#6 and \#12 out of 18 ports, i.e. 120 degrees apart from each other). Both endoscopes will have a coarse viewing system and an external illumination source. Illuminating and collected light will be guided by optical fibres. The fine viewing endoscope will use an imaging fibre bundle consisting of 30.000 fibres allowing an optical resolution of 30um within a 4mm spot. This resolution can be improved by reduction of the $4 \mathrm{~mm}$ field of view.

According to the current conceptual design, each will consist of a shielded enclosure with the endoscope and a push-pull mechanisms inside, a shielded unit for endoscope cleaning and dust sample storage, and a guide tube selector - everything is mounted onto an endoscope trolley inside the Port Cell (Figure 1). The endoscope pipe from the port Cell region is pushed through guide tubes and collects dust on and under the divertor. Guide tubes will be used for control of endoscope routing to the area of interest near the divertor. The guide tubes will have equally spaced holes to allow for dust to deposit inside the tube. Such a structure would provide a better control of the endoscope motion towards the inspection region than simple U-shape tubes and would decrease the risk of jamming. 


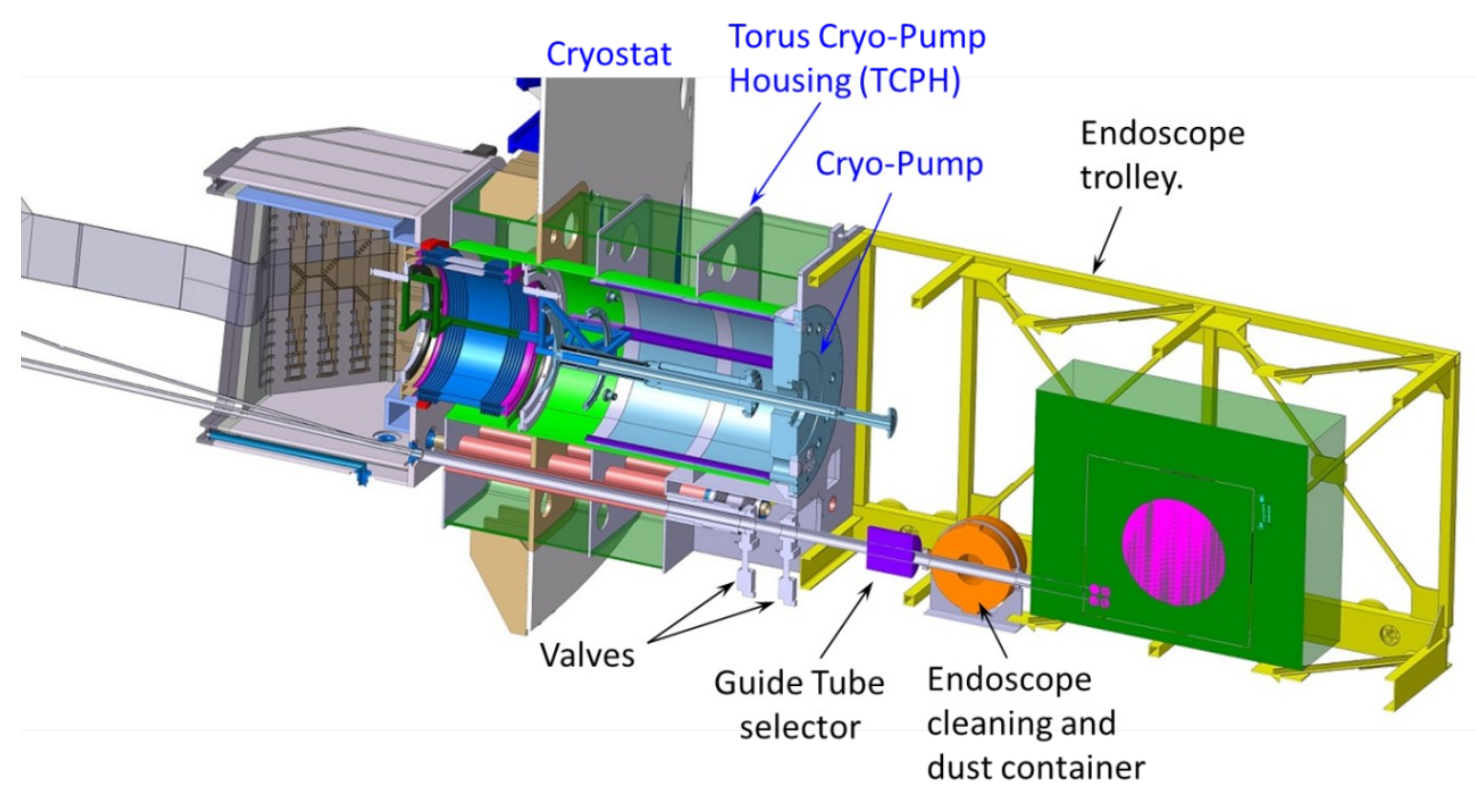

Figure 1. Layout of endoscope enclosure in lower port cell and main interface boundaries.

It is expected that the level of dust produced over a typical experimental campaign of 7000 $\mathrm{Q}=10$ discharges is going to stay well below the safety limit [3]. Most of the dust will be produced during major events such as Vertical Displacement Events, Major Disruptions etc.

As a result, the endoscope will operate on demand, e.g. after some major event or during the short term maintenance period planned once a fortnight. At the same time, there is no history of endoscopes operating in vacuum. Operation of the endoscope in vacuum induces a risk of jamming inside tokamak due to increased friction. Overcoming this issue would require a considerable development effort and the development of appropriate recovery measures in case of endoscope jamming inside the vacuum vessel. To minimize this risk, the tokamak will be vented with dry nitrogen and controlled level of oxygen before endoscope invasion to VV. Nonetheless, jamming is one of the main risks for this diagnostic and a range of tests and precautions are foreseen to avoid it, e.g. estimation of maximum expected friction force, development of appropriate push/pull mechanism, coating of guide tube inner surfaces, development of a rescue scenario, etc. Since the design of Dust Monitor needs to comply with ITER baseline requirements ensuring confinement and radiation protection safety functions, the jamming risk needs to be solved before final acceptance of the diagnostic. This will require demonstration of diagnostic safe performance in all situations.

Since the endoscope will make a survey in a dusty environment with dust size from nanometer to few hundred micrometers, some of the dust may stick to the endoscope jacket. Dust is activated up to few hundred Gy/h contact dose and contamination of the jacket will lead to fibre bundle degradation, as well as represent a risk for personnel working close to the enclosure. To avoid this, the diagnostic will have a cleaning unit to scrub the endoscope pipe during the retraction process. In addition, the jacket of endoscope will be smooth to prevent dust penetration and to simplify cleaning. This prevents the use of braided and similar armoured pipes.

Dust collection is one of the most important functions of endoscope. The collected dust will be transferred to the hot cell facilities and will be analyzed to determine the tritium content, 
size distribution and composition of the dust. The analysis strategy is currently being developed. Different sampling systems are being investigated for the endoscope front-end :

- Gas blow or pumping

- Brush (rotating or reciprocating movement)

- Electrostatic collector

- Sticky pads

- Extractable dust traps (boats)

- Etc.

The use of sticky pads was eliminated from consideration since its usage on regular basis along guide tubes will lead to degradation of primary vacuum of tokamak with increased risk of endoscope jamming inside guide tubes (some fraction of sticky material might stay inside guide tube), moreover in the region of dust accumulation the procedure of dust collection by sticky pads will have to be repeated several times to collect all of the dust. So was the option of extractable dust traps or "boats" with collected dust inside due to concerns on reliability and complexity of such a system. The two main options of dust collection under consideration are an electrostatic collector and a combination of gas blow with a brush. Both require R\&D and demonstration of proof of principle. Notably, gas blow technique for dust collection was already applied in Tore Supra in air, albeit for carbon dust [4]. Proof of principle for electrostatic collection of metallic dust from metallic surface was recently demonstrated in laboratory experiments at MEPhI [5] for W dust. In order to improve the efficiency of dust collection and make a final choice between the different sampling techniques, a better understanding of $\mathrm{W}$ and Be dust adhesion on surfaces is needed. Studies of dust adhesion and remobilization (by plasma impact) have recently been reported for tungsten dust [6]. Since dust in ITER is expected to be composed mainly of beryllium, those studies would need to be extended to beryllium.

\section{Conclusion.}

Endoscope based techniques for dust monitoring will allow on-demand and regular survey under divertor cassettes and extraction of dust for analysis in laboratory. Options for dust collection are based on electrostatic collector or gas blow and brush based collector, both of which require testing to demonstrate proof of principle in various conditions. Jamming of endoscope inside guide tube during inspection was identified as the main risk and requires mitigation strategy focused on design, analysis and testing of guide tubes, with endoscope pipe and sampling head and push/pull mechanisms.

Disclaimer: The views and opinions expressed herein do not necessarily reflect those of the ITER Organization. 


\section{References}

1 Chang-Hwan Choi, Alessandro Tesini, et.al., "Multi-Purpose Deployer for ITER In-Vessel Maintenance," 12th Intrenational Symposium on Fusion Technology, P3 110, 2014

2 G. Dubus, A. Puiu, P. Bates, C. Damiani, R. Reichle, J. Palmer, Progress in the design and R\&D of the ITER In-Vessel Viewing and Metrology System (IVVS), Fusion Engineering and Design 89 (2014) 2398-2403

3 M.Shimada, R.Pitts, et.al. J.Nucl.Mater., 2013, 438, S996-S1000

$4 \quad$ F. Gensdarmes et al, Fus. Eng. end Design 88 (2013) 2684

5 L. Begrambekov, P. Shigin, Private Communications, Moscow, Russia, 2015

$6 \quad$ P. Tolias et al, Plasma Physics and Controlled Fusion, submitted 